\title{
Is orchiectomy avoidable in testicular tuberculosis mimicking malignancy? A case report
}

Youssef Kharbach $^{1,2^{*}}$ (D) Youssef Retal ${ }^{1}$ (D) and Abdelhak Khallouk K $^{1,2}$

\begin{abstract}
Background: Isolated testicular tuberculosis (TB) is extremely uncommon. It has non-specific presentation; thus, diagnosis is challenging and is often discovered on pathology examination after orchiectomy.

Case presentation: We report herein the case of a 73-year-old male, with no significant medical or family history, who presented with left scrotal swelling, physical examination revealed a left testicular firm mass measuring $3 \mathrm{~cm}$ and ultrasound was suggestive of testicular tumor. Left inguinal orchiectomy was performed and the pathologic examination revealed testicular TB. The presentation was typically mimicking a testicular cancer with no evocative evidence of TB; this can lead to a dilemma and highlights the need to consider TB in differential diagnosis of testicular tumor, especially in areas endemic for the disease.
\end{abstract}

Conclusions: The aim of our presentation is to argue if orchiectomy was avoidable. It also illustrates the probable hematogenous or lymphatic spread of Mtb to the testicle.

Keywords: Genitourinary tuberculosis, Testicular tuberculosis, Testicular tumor, Orchiectomy

\section{Background}

Tuberculosis (TB) is an infectious disease caused by bacilli of the Mycobacterium tuberculosis (Mtb) complex $[1,2]$. It has been considered a serious global public health emergency for the past 25 years [1].

Urogenital tuberculosis is the second most common form of extrapulmonary TB [3], but isolated testicular $\mathrm{TB}$, as presented in our patient, is extremely uncommon $[3,4]$. Moreover, the mechanism of spread of TB bacilli to the testis is still controversial [4]. Due to non-specific presentation of testicular TB, diagnosis is challenging and is often discovered on pathology examination after orchiectomy [2].

We report herein the case of an isolated testicular TB mimicking malignancy who had radical orchiectomy. The

\footnotetext{
*Correspondence: ykharbach@uae.ac.ma

${ }^{1}$ Faculty of Medicine and Pharmacy, Abdelmalek Essaâdi University, 90000 Tangier, Morocco

Full list of author information is available at the end of the article
}

aim of our presentation is to argue if orchiectomy was avoidable. It also illustrates the probable hematogenous or lymphatic spread of Mtb to the testicle.

\section{Case presentation}

A 73-year-old Moroccan man presented to our tertiary referral hospital after one week of left scrotal swelling. The patient had no history of tuberculosis or tubercular contact, no significant medical history, he denied trauma and other symptoms.

Physical examination found a left testicular firm mass measuring approximately $3 \mathrm{~cm}$, with irregular surface; scrotal skin and epididymis were normal on palpation. There was no palpable inguinal lymphadenopathy.

Serum tumor markers were within normal limits, AFP: $3.3 \mathrm{ng} / \mathrm{mL}$; HCG:<2 mUI/mL; LDH: 203 U/L. Serology for human immunodeficiency virus (HIV) was negative.

Chest X-ray was within normal limits. Scrotal ultrasound of the left testis revealed a heterogeneous, hypoechoic anterolateral mass measuring $28.9 \times 14.7 \mathrm{~mm}$ 
in diameter (Fig. 1), with internal vascularity on color Doppler imaging suggesting testicular tumor (Fig. 2). Right testis and both epididymes were normal.

In light of these findings, left-sided high inguinal orchiectomy was performed (Fig. 3) after an informed consent due to the preliminary diagnosis of testicular tumor.

Histopathological examination of testicular tissue revealed the presence of large areas of tuberculotic granuloma caseous necrosis (Fig. 4), multinucleated Langhans giant cells were present in the center of granuloma (Fig. 5), and the epididymis was histologically normal. There was no evidence of malignancy.

Acid-fast bacilli (AFB) sputum smear and cultures were negative. Urine smear for AFB was negative, and uroscan found no evidence of abnormality or urinary disorder, thereby eliminating an active site of genitourinary tuberculosis. Based on above findings, the patient was diagnosed as having isolated testicular TB.

The patient was referred at the local tuberculosis treatment center. Anti-tuberculosis chemotherapy was started for 6 months.

\section{Discussion}

Tuberculosis continues to cause considerable morbidity and mortality globally and is considered as a disease of poverty [1]. Risk factors for TB include malnutrition, HIV infection, diabetes, substance abuse, poor housing, smoking, immunosuppressive drugs, and chronic renal disease $[1,2]$.

TB generally affects the entire male genital tract [3]. Prostate and epididymis tuberculosis occurs through hematogenous spread [3]. Genital TB can also occur through the urinary system to the prostate, and then, it spreads from the ejaculatory ducts to the seminal vesicles, vas deferens, and epididymis [3]. The testicles are affected by contiguity with the epididymis, because the blood-testicles barrier plays a protective role [3]. However, this mechanism of spread of TB bacilli to the testis is controversial. Some reported cases of isolated testicular tuberculosis and our case suggest that patients may develop isolated testicular TB through hematogeneous or/and lymphatic spread [4]. Hence, isolated testicular TB with no epididymal involvement is very rare, which we present in our case report.

The diagnosis of urogenital tuberculosis is presumptive and based on a patchwork of suggestive clinical,

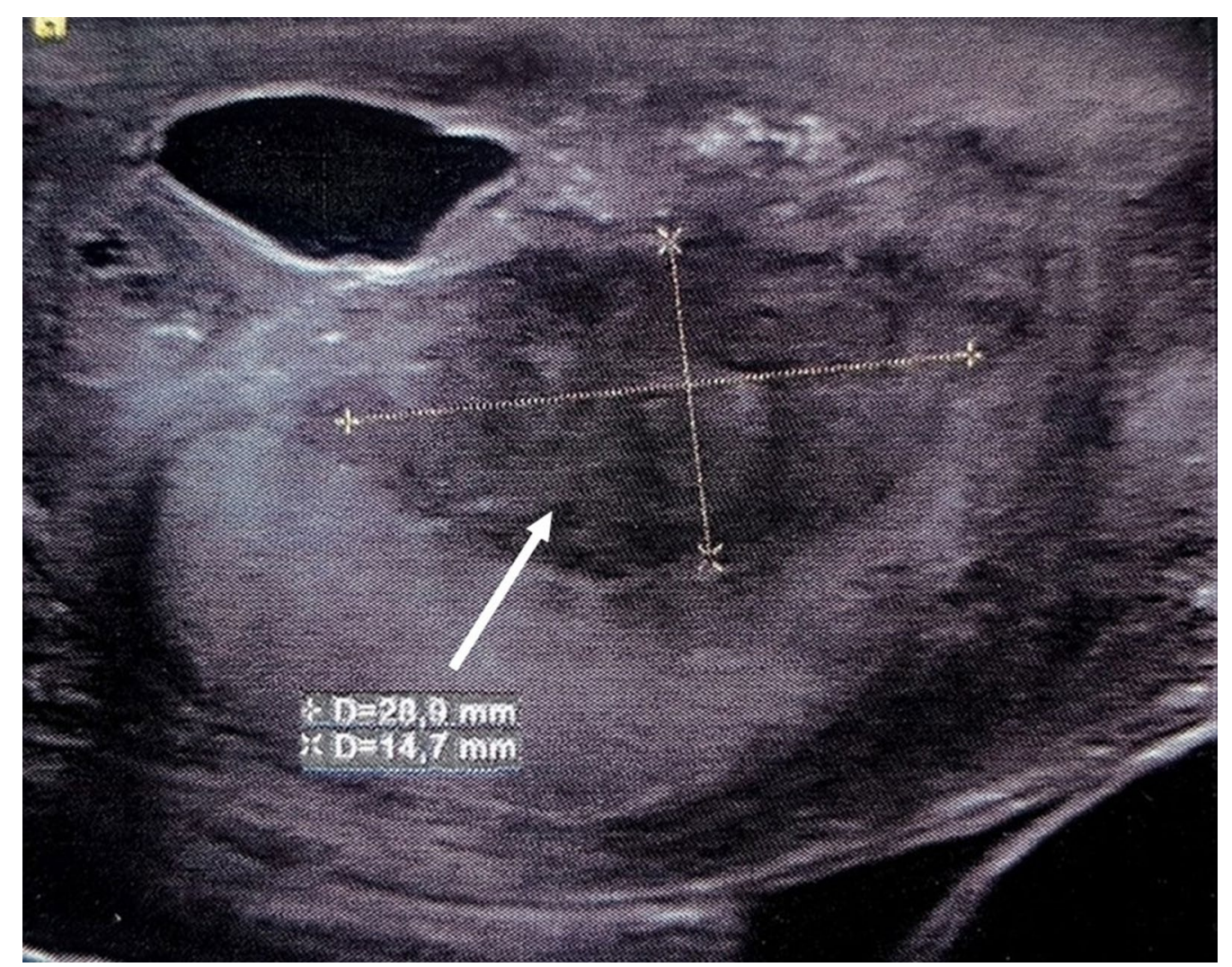

Fig. 1 Ultrasonography of left testis showing a heterogeneous and hypoechoic mass measuring $28.9 \times 14.7 \mathrm{~mm}$ in diameter 


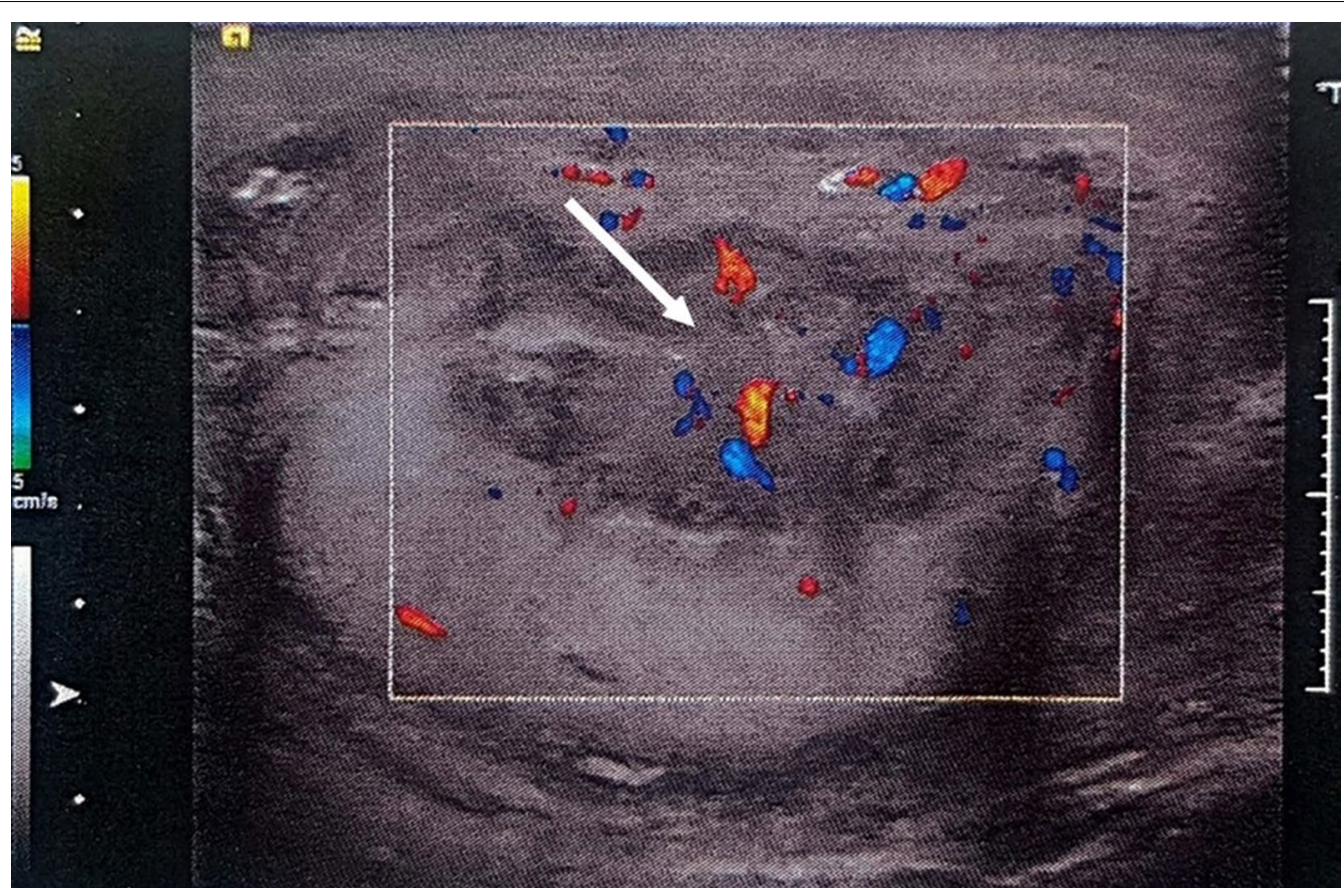

Fig. 2 Color Doppler image showing internal vascularity of the testicular mass

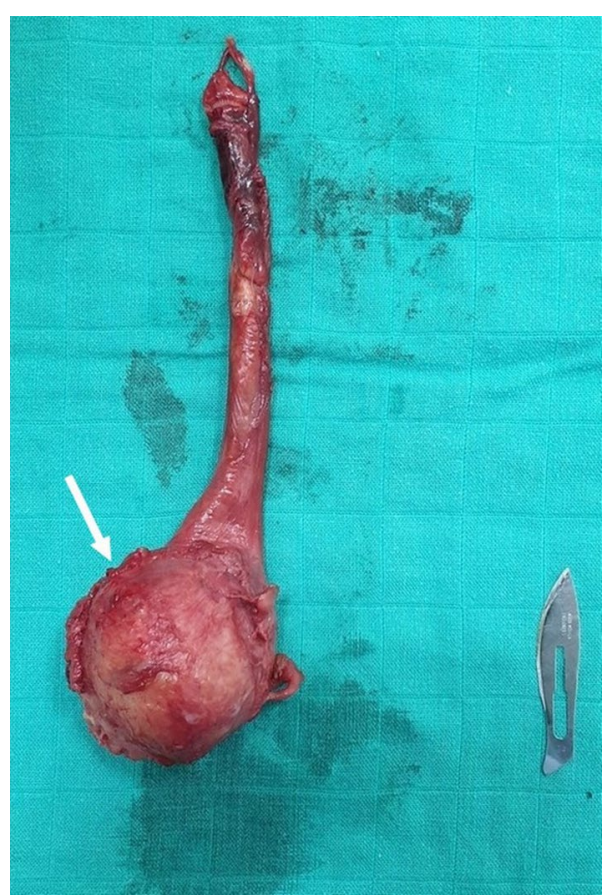

Fig. 3 Postoperative picture of orchiectomy

biological and radiological arguments, without microbiological or histological confirmation in approximately

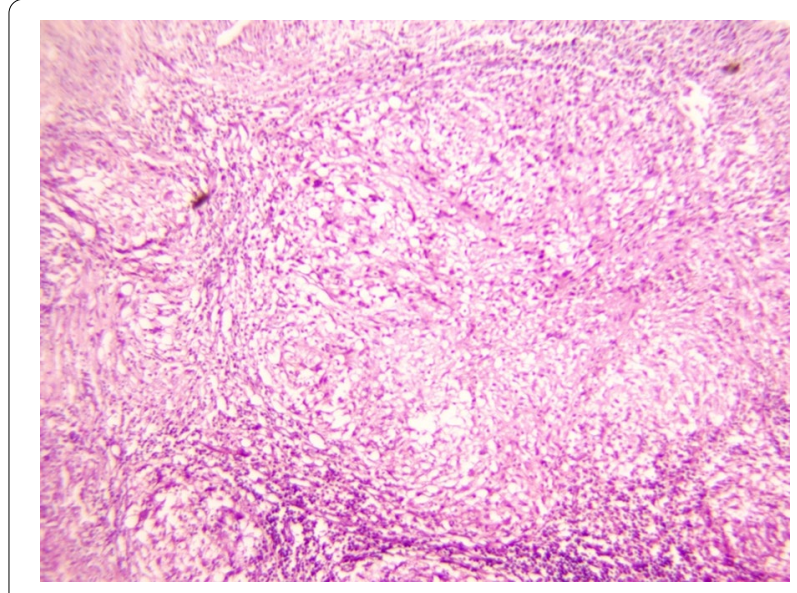

Fig. 4 Photomicrograph showing granulomatous inflammation in testicular tissue, featuring caseous necrosis (H\&E stain, $\times 100$ )

$10.4 \%$ of patients $[3,5]$. Subsequently, diagnosis of epididymo-testicular tuberculosis is challenging and is discovered on pathology examination after orchidectomy in up to one fifth of cases [2].

Non-specific constitutional symptoms of TB such as fever, weight loss, and night sweats are uncommon in testicular TB [2]. Patient could present acute or chronic, painful, or painless scrotal swellings [2]. Physical examination can find a non-tender testicular mass 


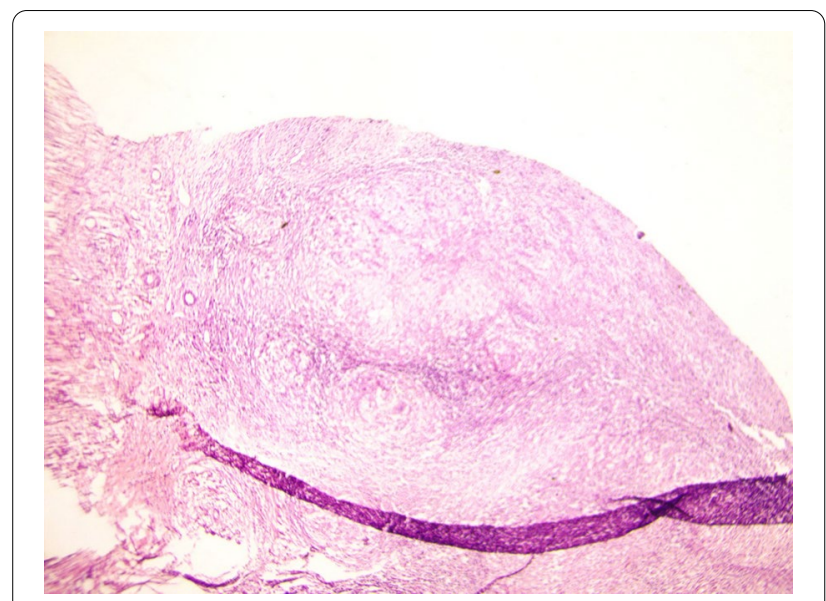

Fig. 5 Photomicrograph showing epitheloid granuloma with multinucleated Langhans-type giant cells (H\&E stain, $\times 40$ )

[2]. Associated scrotal skin inflammation and fistulae discharging pus are suggestive of TB [2].

Ultrasound examination is useful, but it is non-specific [4]. It can show various patterns, depending on the pathologic stage of tubercular infection, including diffusely enlarged heterogeneously hypoechoic testis, diffusely enlarged homogeneously hypoechoic testis, and nodular enlarged heterogeneously hypoechoic testis [6]. Smooth peripheral calcification of tunica vaginalis (typical of TB), concurrent involvement of the epididymis and scrotal wall thickening are highly suggestive of testicular TB [7]. Color Doppler can help differentiate testicular TB from tumor in which generally shows central vascularity, whereas TB demonstrates peripheral vascularity due to granulomas and lack of central flow due to caseation necrosis $[7,8]$.

Microbiological diagnostic method for TB is usually not helpful in testicular TB. Smear microscopy diagnostic yields using urine are below 40\% [2]. Culture in Lowenstein-Jensen medium is the diagnostic gold standard for urogenital tuberculosis [3], but it is not contributory in isolated testicular TB. PCR for Mtb identification in the urine is highly sensitive and specific and may contribute to the diagnosis [3].

In our case, the patient's presentation with only painful scrotal swelling and ultrasound findings prompted the diagnosis of testicular cancer even with the normal level of serum tumor markers suggesting non-seminomatous germ cell tumor of the testis for example. Fine-needle aspiration biopsy (FNAB) was not done to avoid causing local spread of tumor cells or to the inguinal lymph nodes.

Actually, FNAB is usually prohibited in testicles masses because of scrotal violation that can lead to neoplastic cell dissemination $[9,10]$. FNAB is especially helpful in young patients with testicular swelling and normal testicular tumor markers [10], especially if epidemiologic risk factors for TB are present [11].

Treatment consists of four drugs (isoniazid, rifampicin, pyrazinamide, and ethambutol) given for a total of 2 months followed by two drugs (isoniazid and rifampicin) given for an additional 4 months [1].

\section{Conclusions}

Testicular TB is a curable disease, but its diagnosis remains challenging. It is often missed owing to its nonspecific symptoms. Thus, testicular TB should be suspected in patients with a notion of contagion or history of tuberculosis. Some ultrasound features are highly suggestive of TB.

FNAB could prevent unnecessary orchiectomy, but there is a lack of consensus on its use.

In our case, the presentation was typically mimicking a testicular cancer with no evocative evidence of TB. It seems to us that orchiectomy was not avoidable in this patient.

\section{Abbreviations}

TB: Tuberculosis; Mtb: Mycobacterium tuberculosis; HIV: Human immunodeficiency virus; AFB: Acid-fast bacilli; FNAB: Fine-needle aspiration biopsy.

\section{Acknowledgements}

Not applicable.

\section{Authors' contributions}

YK and AK were involved in concept, design, supervision, processing, writing the manuscript and critical analysis. AK revised the manuscript. YR was involved in data acquisition. All authors read and approved the final manuscript.

Funding

This research received no specific Grant from any funding agency in the public, commercial, or not-for-profit sectors.

\section{Availability of data and materials}

The datasets generated during and/or analyzed during the current study are available from the corresponding author on reasonable request.

\section{Declarations}

\section{Ethics approval and consent to participate}

Tangier University Hospital does not require ethical approval for reporting individual cases or case series.

\section{Consent for publication}

Written informed consent was obtained from the patient for publication of this case report and any accompanying images.

\section{Competing interests}

The authors report no competing personal or financial interest related to this work. 


\section{Author details}

${ }^{1}$ Faculty of Medicine and Pharmacy, Abdelmalek Essaâdi University, 90000 Tangier, Morocco. ${ }^{2}$ Urology Department, Tangier-Tetouan-Alhoceima University Hospital, 90000 Tangier, Morocco.

Received: 5 June 2021 Accepted: 19 September 2021

Published online: 11 October 2021

\section{References}

1. Furin J, Cox H, Pai M (2019) Tuberculosis. Lancet (London, England) 393:1642-1656

2. Muneer A, Macrae B, Krishnamoorthy S et al (2019) Urogenital tuberculosis-epidemiology, pathogenesis and clinical features. Nat Rev Urol 16:573-598

3. Figueiredo AA, Lucon AM, Srougi M (2017) Urogenital tuberculosis. Microbiol Spectr. https://doi.org/10.1128/microbiolspec.TNMI7-0015-2016

4. Agbo CA, Lawal M, Jibrin D et al (2020) An isolated testicular tuberculosis mimicking testicular cancer in north-central Nigeria. Infez Med 28:78-81

5. Figueiredo AA, Lucon AM, Junior RF et al (2008) Epidemiology of urogenital tuberculosis worldwide. Int J Urol 15:827-832
6. Muttarak M, Peh WC, Lojanapiwat B et al (2001) Tuberculous epididymitis and epididymo-orchitis: sonographic appearances. AJR Am J Roentgenol 176:1459-1466

7. Nepal P, Ojili V, Songmen S et al (2019) "The Great Masquerader": sonographic pictorial review of testicular tuberculosis and its mimics. J Clin Imaging Sci 9:27

8. Yang DM, Chang MS, Oh YH et al (2000) Chronic tuberculous epididymitis: color Doppler US findings with histopathologic correlation. Abdom Imaging 25:559-562

9. Assi A, Patetta R, Fava C et al (2000) Fine-needle aspiration of testicular lesions: report of 17 cases. Diagn Cytopathol 23:388-392

10. Ratkal V, Chawla A, Mishra DK et al (2015) Testicular non-Hodgkin's lymphoma presenting in a young adult. BMJ Case Rep 2015:bcr2014208633

11. Sharma A, Nagalli S, Varughese AT et al (2020) A review of the diagnostic use of fine-needle aspiration cytology for tuberculosis epididymo-orchitis: to do or not to do. Cureus 12:e6532

\section{Publisher's Note}

Springer Nature remains neutral with regard to jurisdictional claims in published maps and institutional affiliations.

\section{Submit your manuscript to a SpringerOpen ${ }^{\circ}$ journal and benefit from:}

- Convenient online submission

- Rigorous peer review

- Open access: articles freely available online

- High visibility within the field

- Retaining the copyright to your article

Submit your next manuscript at $\gg$ springeropen.com 